order small quantities even in the compressible case. The major effect of the vertical velocity component in the determination of the stability boundary is through $x_{31}^{(1)}$ and $x_{32}^{(1)}$.

Suppose we take all $x_{3 i}$ from the $\epsilon$ series and consistently take all six solutions to the order of $\epsilon$ i.e. $x_{3 i}^{(0)}+\epsilon x_{3 i}^{(1)}$ in the boundary value problem, the $v$-dependence of $x_{31}^{(1)}$ and $x_{32}^{(1)}$ indicates an inconsistency of the simplification of assuming the boundary layer flow as parallel flow. Fortunately, all the previous investigators are satisfied with the first approximation of the two viscous solutions $x_{31}^{(0)}$ and $x_{32}^{(0)}$ while they used $x_{33}^{(0)}+$ $\epsilon x_{33}^{(1)}$ and $x_{34}^{(0)}+\epsilon x_{34}^{(1)}$ or the two equivalent inviscid solutions or the inviscid solutions corrected for viscosity in the boundary value problems. Hence, the stability boundary as determined by any of these methods is independent of $v$ and their results are consistent with the assumption that boundary layer flows are parallel flows. Therefore, within the order of approximation attempted by previous investigators, the stability of the laminar boundary layer is determined only by the local flow properties for both the compressible and the incompressible flow.

The accuracy of the quantitative determination of the stability boundary as carried out in references 2,3 and 4, however, can not be improved merely by taking more terms in the $\epsilon$ series without including the effect of the vertical velocity component. It is unfortunate that, in some practical cases, the parameter $\epsilon$ may be only 0.1 near the minimum critical Reynolds number and as such the second approximation of the order of $\epsilon$ should better be considered for accurate determination of the stability limit and the initial amplification rate, in which cases, the effect of the vertical velocity component must be included.

In addition, at high Mach numbers, the vertical velocity component in the boundary layer is of the order of $M^{4} / R$, and may enter the stability problem of the laminar boundary layer even in the first approximation. The stability of the hypersonic laminar boundary layer, therefore, requires careful investigation.

\title{
REFERENCES
}

1. L. Lees and C. C. Lin, Investigation of the stability of the laminar boundary layer in a compressible fluid, N.A.C.A. T.N.1115 (1946).

2. L. Lees, The stability of laminar boundary layer in a compressible fluid, N.A.C.A. T.N. 1360 (1947).

3. J. Pretsch, Die Stabilität einer ebenen Laminarströmung bei Druckgefälle und Druckanstieg, Jahrbuch der Deutschen Luftfahrtforschung, 1, 58-75 (1941).

4. J. A. Laurmann, Stability of compressible laminar boundary layer with pressure gradient, College of Aeronautics, Cranfield, England, Report No. 48, (1951).

\section{STRESS-STRAIN RELATIONS, UNIQUENESS AND VARIATIONAL THEOREMS FOR ELASTIC-PLASTIC MATERIALS WITH A SINGULAR YIELD SURFACE*}

\author{
By W. T. KOITER (Technical University, Delft, Holland)
}

1. Plastic stress-strain relations. The state of stress at any point of a continuous medium is described by the stress tensor $\sigma_{i i}$ and may be represented by a point in ninedimensional stress space. It will be assumed that no yielding occurs if the stress point

*Received Dec. 22, 1952. 
lies within a convex domain in stress space, which will be called the elastic domain. The boundary of the elastic domain is the yield surface which is called regular if it is described by an equation

$$
f\left(\sigma_{i j}\right)=0,
$$

where $f$ is a regular (continuously differentiable) function, the yield function, of its nine variables, symmetrical with respect to $\sigma_{i j}$ and $\sigma_{i i}$. The sign of $f$ is chosen such that $f\left(\sigma_{i j}\right)$ is negative in the elastic domain. For a so-called perfectly plastic material that yields under constant stresses, Prager [1] has shown under some very general assumptions that the plastic strain rate tensor is given by

$$
\epsilon_{i i}^{\prime \prime \prime}=\lambda \frac{\partial f}{\partial \sigma_{i j}},
$$

where

$$
\left.\begin{array}{lccc}
\lambda=0 & \text { for } & f<0 & \\
& \text { and also for } & f=0, & f^{\cdot} \equiv \frac{\partial f}{\partial \sigma_{i i}} \sigma_{i i}<0 ; \\
\lambda \geq 0 & \text { for } & f=0, & f^{\cdot} \equiv \frac{\partial f}{\partial \sigma_{i i}} \sigma_{i j}=0 .
\end{array}\right\}
$$

For a material with strain-hardening the relations (2) and (3) are replaced by

$$
\left.\begin{array}{cccc}
\dot{\epsilon}_{i i}^{\prime \prime}=0 & \text { for } & f<0 & \\
& \text { and also for } & f=0, & f^{\prime} \equiv \frac{\partial f}{\partial \sigma_{i j}} \sigma_{i i} \leq 0 ; \\
\epsilon_{i i}^{\prime \prime \prime}=h \frac{\partial f}{\partial \sigma_{i j}} f^{.} & \text {for } & f=0, & f^{\cdot} \equiv \frac{\partial f}{\partial \sigma_{i j}} \sigma_{i i} \geq 0,
\end{array}\right\}
$$

where $h$ is a scalar function of stress, plastic strain and strain history. The plastic stressstrain relations (2), (3) and (4) may be called associated with the yield function. If the plastic strain rate is depicted in nine-dimensional stress space, these relations are expressed by the geometrical statement that the direction of the plastic strain rate is given by the normal to the yield surface.

In this paper singular yield surfaces will be considered of a type described by a number of regular yield functions $f_{p}\left(\sigma_{i j}\right)$ (symmetrical with respect to $\sigma_{i i}$ and $\sigma_{i i}$ ) such that the elastic domain is given by

$$
f_{p}\left(\sigma_{i j}\right)<0 \quad p=1,2, \cdots n
$$

and that yielding occurs as soon as at least one of the functions $f_{p}$ is zero. All points of the yield surface, where only one function $f_{p}=0$, are regular and the corresponding plastic strain rates are the same as for a completely regular yield surface $f_{p}=0$. Difficulties arise only on the intersection of two or more surfaces $f_{p}=0$ because for these stress states the normal to the yield surface becomes indeterminate. However, this ambiguity is largely removed if the criterion for unloading or yielding at such a singular 
point is applied for each yield function separately. Physically this assurnption is entirely plausible because each yield function represents a separate yield criterion. Its mathematical expression is for a perfectly plastic material

$$
\epsilon_{i j}^{\prime \prime \prime}=\sum_{p=1}^{n} \lambda_{p} \frac{\partial f_{p}}{\partial \sigma_{i j}}
$$

where

$$
\left.\begin{array}{lccc}
\lambda_{p}=0 & \text { for } & f_{p}<0, & \\
& \text { and also for } & f_{p}=0, & f_{p}^{\prime} \equiv \frac{\partial f_{p}}{\partial \sigma_{i i}} \sigma_{i j}<0 ; \\
\lambda_{p} \geq 0 & \text { for } & f_{p}=0, & f_{p}^{*} \equiv \frac{\partial f_{p}}{\partial \sigma_{i j}} \sigma_{i i}=0
\end{array}\right\}
$$

For a material with strain-hardening the relations (6) and (7) are replaced by

$$
\epsilon_{i i}^{\prime \prime \prime}=\sum_{p=1}^{n} c_{p} h_{p} \frac{\partial f_{p}}{\partial \sigma_{i j}} f_{p}^{.}
$$

where $h_{p}$ is a positive function of stress, plastic strain and strain history, and $c_{p}$ is zero if either $f_{p}<0$ or $f_{p}^{*} \leq 0$ and unity for $f_{p}=0$ and $f_{p}^{*} \geq 0$.

2. The variational and uniqueness theorems. Current proofs of the variational and uniqueness theorems for a material with a regular yield surface and the associated plastic stress-strain relations are all of them based on the inequality (see [2])

$$
\sigma_{i j(1)} \dot{\epsilon}_{i j(1)}+\sigma_{i j(2)} \dot{\epsilon}_{i j(2)}-2 \sigma_{i j(1)} \dot{\epsilon}_{i j(2)} \geq 0 \text {, }
$$

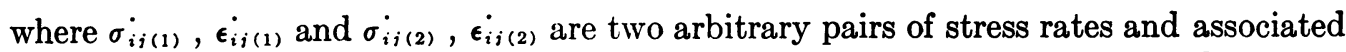
strain rates at the same point of stress space. The equality sign in (9) holds only if $\sigma_{i j(1)}^{-}=\sigma_{i(2)}^{\cdot}$. This inequality is applied directly to prove the two variational theorems, and the uniqueness theorem is shown to hold by means of the inequality, obtained by interchanging the suffixes 1 and 2 in (9) and adding

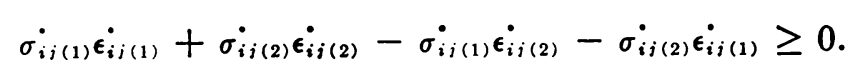

The proof of (9) is obtained by considering the elastic and plastic strain rates separately. Hooke's law leads to the inequality

$$
\sigma_{i i(1)} \dot{\epsilon}_{i j(1)}^{\prime \prime}+\sigma_{i j(2)}^{\cdot \epsilon_{i j(2)}^{\prime \prime}}-2 \sigma_{i j(1)}^{\cdot \epsilon_{i j(2)}^{\prime \prime}} \geq 0,
$$

where $\epsilon_{i j}^{\prime \prime}$ is the elastic strain rate tensor; and the equality sign applies only if $\sigma_{i i(1)}=$ $\sigma_{i j(2)}$. The similar inequality for the plastic strain rates

$$
\sigma_{i j(1)}^{\cdot \epsilon_{i j(1)}^{\prime \prime}}+\sigma_{i i(2)}^{\cdot \epsilon_{i j(2)}^{\prime \prime}}-2 \sigma_{i j(1)}^{\prime \prime} \epsilon_{i j(2)}^{\prime \prime \prime} \geq 0
$$

is shown to hold by means of the stress-strain relations (2), (3) or (4).

It will now be proved that the stress-strain relations (6)-(8) also lead to inequality (12) hence ensure the validity of the uniqueness and variational theorems. For a 
perfectly plastic material the left-hand member of (12) is calculated as follows

$$
\begin{aligned}
& \sigma_{i j(1)}^{\dot{\epsilon_{i j(1)}^{\prime \prime}}}+\sigma_{i j(2)}^{\dot{\epsilon}_{i j(2)}}-2 \sigma_{i j(1)}^{\prime \prime \epsilon_{i j(2)}^{\prime \prime \prime}} \\
& =\sum_{p=1}^{n}\left[\lambda_{p(1)} \sigma_{i i(1)} \frac{\partial f_{p}}{\partial \sigma_{i i}}+\lambda_{p(2)} \dot{\sigma}_{i i(2)}^{\cdot} \frac{\partial f_{p}}{\partial \sigma_{i j}}-2 \lambda_{p(2)} \dot{\sigma}_{i i(1)}^{\cdot} \frac{\partial f_{p}}{\partial \sigma_{i i}}\right] \\
& =\sum_{p=1}^{n}\left[\lambda_{p(1)} f_{p(1)}^{\cdot}+\lambda_{p(2)} f_{p(2)}^{\cdot}-2 \lambda_{p(2)} f_{p(1)}^{\cdot}\right] \text {. }
\end{aligned}
$$

The various possibilities for each term in this sum are listed below with the consequences, ensuing from the stress-strain relations (6), (7):

$$
\begin{aligned}
& f_{p}<0 \rightarrow \lambda_{p(1)}=\lambda_{p(2)}=0, \\
& f_{p}=0, \quad f_{p(1)}^{\cdot}<0, \quad f_{p(2)}^{\cdot}<0 \rightarrow \lambda_{p(1)}=\lambda_{p(2)}=0, \\
& f_{p}=0, \quad f_{p(1)}^{\cdot}=0, \quad f_{p(2)}^{\cdot}<0 \rightarrow \lambda_{p(1)} \geq 0, \quad \lambda_{p(2)}=0, \\
& f_{p}=0, \quad f_{p(1)}^{\cdot}<0, \quad f_{p(2)}^{\cdot}=0 \rightarrow \lambda_{p(1)}=0, \quad \lambda_{p(2)} \geq 0, \\
& f_{p}=0, \quad f_{p(1)}^{\cdot}=0, \quad f_{p(2)}^{\cdot}=0 \rightarrow \lambda_{p(1)} \geq 0, \quad \lambda_{p(2)} \geq 0,
\end{aligned}
$$

It follows that only if (13d) applies a non-zero term occurs and this term is then always positive. The inequality (12) must therefore hold true and the uniqueness and variational theorems are proved.

The proof of (9) for a strain-hardening material with stress-strain reiations (8) is entirely similar and may be omitted here.

3. Application to Tresca's yield criterion for a perfectly plastic material. In some problems, e.g. the thick-walled tube under internal pressure [3], Tresca's yield criterion and its associated stress-strain relations afford a much simpler approach than von Mises' yield condition and its associated Prandtl-Reuss relations. It will now be shown that Tresca's yield criterion belongs to the singular yield criteria considered here.

The most convenient formulation of Tresca's yield criterion for the present purpose is that the shear stress component in any plane, defined by its unit normal $n_{i}^{(a)}$, and in any direction $n_{i}^{(b)}$ in this plane (which is of course perpendicular to $n_{i}^{(a)}$ shall not exceed the yield stress in pure shear. This criterion may be written in a form symmetrical with respect to $\sigma_{i j}$ and $\sigma_{i i}$

$$
\frac{1}{2} \sigma_{i i}\left\{n_{i}^{(a)} n_{1}^{(b)}+n_{i}^{(a)} n_{i}^{(b)}\right\}-k \leq 0,
$$

where $k$ is the yield stress in shear. Clearly the number of yield functions is now infinite but this does not affect the foregoing argument and the uniqueness and variational theorems remain valid. The plastic strain rates associated with the yield functions (14) follow from (6)

$$
\epsilon_{i i}^{\prime \prime}=\sum_{(a, b)} \frac{1}{2} \lambda_{(a b)}\left\{n_{i}^{(a)} n_{i}^{(b)}+n_{i}^{(a)} n_{i}^{(b)}\right\},
$$


where $\lambda_{(a b)}$ is only non-zero for those directions $n_{t}^{(a)}$ and $n_{i}^{(b)}$ for which the equality sign in (14) applies and moreover

$$
\sigma_{i}\left\{n_{i}^{(a)} n_{i}^{(b)}+n_{i}^{(a)} n_{i}^{(b)}\right\}=0 .
$$

It is obvious that the plastic strain rate, associated with a yield function (14), is a pure shear in the directions $n_{i}^{(a)}$ and $n_{i}^{(b)}$.

If the principal stresses are unequal, $\sigma_{1}>\sigma_{2}>\sigma_{3}$, there is only one pair of directions $n_{i}^{(a)}, n_{i}^{(b)}$ for which the equality sign in (14) can hold; this occurs if $\sigma_{1}-\sigma_{3}=2 k$ and the plastic strain rate is a pure shear with its principal axes along the major and minor principal stress axes. If two principal stresses are equal, e.g. $\sigma_{1}>\sigma_{2}=\sigma_{3}$, there is a one-parametric family of pairs of directions $n_{i}^{(a)}, n_{i}^{(b)}$, for which the equality sign in (14) holds. However, if the stress rates are such that $\sigma_{i}^{*}=\sigma_{3}^{+}<\sigma_{2}^{\cdot}$, there is only one pair of directions in this family for which (16) holds true and the plastic strain rate is again a pure shear. On the other hand, if both $\sigma_{1}-2 k=\sigma_{2}=\sigma_{3}$ and $\sigma_{1}^{*}=\sigma_{2}^{*}=\sigma_{3}^{*}$, the plastic strain rate is indeterminate; the stress-strain relations only require that one of the principal axes of the plastic strain rate tensor coincides with the major principal axis of the stress tensor, that the two other principal plastic strain rates are both negative and that the plastic volume strain is zero.

4. The slip theory of Batdorf and Budiansky. Considerable interest has been aroused by the slip theory of plasticity for strain-hardening materials, advanced by Batdorf and Budiansky some years ago [4], partly because it seemed to represent a new approach, entirely different from flow and deformation theories. However, this theory may be regarded as a special case of the present theory for materials with a singular yield surface. The number of regular yield functions, of which the yield condition is composed, is again infinite; the yield functions are similar to (14)

$$
\frac{1}{2} \sigma_{i j}\left\{n_{i}^{(a)} n_{i}^{(b)}+n_{i}^{(a)} n_{i}^{(b)}\right\}-k_{(a b)} \leq 0,
$$

where $k_{(a b)}$ now represents either the initial yield stress in pure shear or (if the latter is higher) the highest previous value of the shear stress component with respect to the two mutually orthogonal directions $n_{i}^{(a)}$ and $n_{i}^{(b)}$. The plastic stress-strain relations of Batdorf and Budiansky may be expressed in the form

$$
\dot{\epsilon}_{i j}^{\prime \prime}=\int c_{(a b)} H_{(a b)} \frac{1}{4}\left\{n_{i}^{(a)} n_{i}^{(b)}+n_{i}^{(a)} n_{i}^{(b)}\right\}\left\{n_{p}^{(a)} n_{a}^{(b)}+n_{a}^{(a)} n_{\nu}^{(b)}\right\} \sigma_{D Q}^{\cdot} d \Omega_{(a b)},
$$

where $d \Omega_{(a b)}$ is the measure of the three-dimensional set of pairs of mutually orthogonal directions $n_{i}^{(a)}, n_{i}^{(b)}$ within an infinitesimal region, $H_{(a b)}$ is the characteristic strain hardening function of slip theory which depends only on $k_{(a b)}$, and $c_{(a b)}$ plays the role of $c_{p}$ in (8). It is easily seen that (18) represents an example of (8) for the case of an infinite number of yield functions $f_{p}$. Consequently the uniqueness and variational theorems are equally applicable to a material that follows the slip theory of plasticity.

\section{REFERENCES}

1. W. Prager, Recent developments in the mathematical theory of plasticity, J. Appl. Phys. 20, 235 (1949).

2. R. Hill, Mathematical theory of plasticity, Oxford, 1950.

3. W. T. Koiter, On partially plastic thick-walled tubes, C. B. Biezeno Anniversary Volume, H. Stam, Haarlem, 1953, p. 232.

4. S. B. Batdorf and B. Budiansky, A mathematical theory of plasticity based on the concept of slip, NACA Techn. Note 1871 (1949). 